\title{
ENUMERATION OF QUARTIC FIELDS OF SMALL DISCRIMINANT
}

\author{
JOHANNES BUCHMANN, DAVID FORD, AND MICHAEL POHST
}

\begin{abstract}
With the mixed-type case now completed, all algebraic number fields of degree 4 with absolute discriminant $<10^{6}$ have been enumerated. Methods from the totally real and totally complex cases were used without major modification. Isomorphism of fields was determined by a method similar to one of Lenstra. The $T_{2}$ criterion of Pohst was applied to reduce the number of redundant examples.
\end{abstract}

\section{INTRODUCTION}

We have previously enumerated totally real (signature 4) [1] and totally complex (signature 0) [2] fields. We now treat the remaining case, fields of mixed type (signature 2). The methods of [1] and [2] are used without major modification.

\section{EXISTENCE OF SMALL INTEGERS}

A consequence of $[8$, Theorems $1-3]$ is

Proposition 1. If $F$ is an algebraic number field of degree 4 with discriminant $d_{F}$ and ring of integers $\mathscr{O}_{F}$, then there exists $\rho \in \mathscr{O}_{F} \backslash \mathbb{Z}$ such that

$$
T_{2}(\rho) \leq 1+\sqrt[3]{\left|d_{F}\right| / 2},
$$

where $\rho_{1}, \ldots, \rho_{4}$ are the conjugates of $\rho$, and, for $k \in \mathbb{Z}$,

$$
T_{k}(\rho)=\sum_{j=1}^{4}\left|\rho_{j}\right|^{k}
$$

We let $\rho$ be an algebraic number satisfying the conditions of Proposition 1, with characteristic polynomial given by

$$
f(x)=\prod_{j=1}^{4}\left(x-\rho_{j}\right)=x^{4}-s x^{3}+p x^{2}-q x+n .
$$

If $\mathbb{Q}(\rho)$ has signature 2, then $\rho$ may be assumed to satisfy the following:

$$
\begin{aligned}
0 & \leq s \leq 2, \\
\frac{s^{2}-T_{2}(\rho)}{2} & \leq p \leq \frac{s^{2} / 2+T_{2}(\rho)}{2},
\end{aligned}
$$

Received by the editor November 5, 1990 and, in revised form, June 15, 1992.

1991 Mathematics Subject Classification. Primary 11-04, 11R16, 11Y40.

The second author's research was supported by the Natural Sciences and Engineering Research Council (Canada) and Fonds pour la Formation de Chercheurs et l'Aide à la Recherche (Québec). 


$$
\begin{aligned}
\frac{-s^{3}+3 s p-T_{3}(\rho)}{3} & \leq q \leq \frac{-s^{3}+3 s p+T_{3}(\rho)}{3}, \\
0 & \leq n \leq \frac{T_{2}^{2}(\rho)}{16} .
\end{aligned}
$$

Relations (1), (2), and (4) come directly from [8, Theorem 3], while (3) is a consequence of $\left|s^{3}-3 s p+3 q\right|=\left|\sum_{j=1}^{4} \rho_{j}^{3}\right| \leq T_{3}(\rho)$. We take

$$
\begin{array}{ll}
\widehat{T}_{21}(d)=\lfloor 1+\sqrt[3]{|d| / 2}\rfloor, & \widehat{T}_{3}(d)=\left\lfloor(1+\sqrt[3]{|d| / 2})^{3 / 2}\right\rfloor, \\
\widehat{T}_{22}(d)=\lfloor 2(1+\sqrt[3]{|d| / 2})\rfloor, & \widehat{T}_{4}(d)=\left\lfloor(1+\sqrt[3]{|d| / 2})^{2}\right\rfloor,
\end{array}
$$

and define $G_{1}(d)$ to be the set of all polynomials $f(x)=x^{4}-s x^{3}+p x^{2}-q x+n \in$ $\mathbb{Z}[x]$ with coefficients satisfying

$$
\begin{aligned}
0 & \leq s \leq 2, \\
\frac{s^{2}-\widehat{T}_{21}(d)}{2} & \leq p \leq \frac{s^{2}+\widehat{T}_{22}(d)}{4}, \\
\frac{-s^{3}+3 s p-\widehat{T}_{3}(d)}{3} & \leq q \leq \frac{-s^{3}+3 s p+\widehat{T}_{3}(d)}{3}, \\
0 & \leq n \leq \frac{\widehat{T}_{4}(d)}{16} .
\end{aligned}
$$

The integer quantities $\widehat{T}_{21}(d), \widehat{T}_{22}(d), \widehat{T}_{3}(d), \widehat{T}_{4}(d)$ are easy to compute, so the question of whether a given polynomial belongs to $G_{1}(d)$ can be answered quickly.

Observing that $\left\lfloor T_{2}(\rho)\right\rfloor \leq \widehat{T}_{21}\left(d_{F}\right),\left\lfloor 2 T_{2}(\rho)\right\rfloor \leq \widehat{T}_{22}\left(d_{F}\right),\left\lfloor T_{3}(\rho)\right\rfloor \leq\left\lfloor T_{2}(\rho)^{3 / 2}\right\rfloor$ $\leq \widehat{T}_{3}\left(d_{F}\right),\left\lfloor T_{2}^{2}(\rho)\right\rfloor \leq \widehat{T}_{4}\left(d_{F}\right)$ establishes

Proposition 2. If $F$ is an algebraic number field of degree 4 and signature 2 with discriminant $d_{F}$ and ring of integers $\mathscr{O}_{F}$, then there exists $\rho \in \mathscr{O}_{F} \backslash \mathbb{Z}$ with characteristic polynomial belonging to $G_{1}\left(d_{F}\right)$.

\section{FieldS With SMALl QUARTIC INTEGERS}

For each polynomial $f(x) \in G_{1}\left(10^{6}\right)$ we perform the following tests (cf. [1]):

1. If $f(x)$ is reducible in $\mathbb{Z}[x]$, we exclude $f$.

2. If $f(x)$ is irreducible but $\mathbb{Q}(\rho)$ is not of signature 2 , we exclude $f$.

3. We compute the discriminant $d_{F}$ of the field $F=\mathbb{Q}(\rho)$.

4. If $\left|d_{F}\right| \geq 10^{6}$, we exclude $f$.

5. If $f \notin G_{1}\left(d_{F}\right)$, we exclude $f$.

Among the polynomials surviving these tests is a generating polynomial for every quartic number field of mixed type with absolute discriminant less than $10^{6}$, excepting those fields for which no choice of $\rho$ in Proposition 1 gives a quartic integer. In these fields every such "small" $\rho$ generates a quadratic subfield; we enumerate these fields separately. 


\section{FIELDS WITH QUADRATIC SUBFIELDS}

We now assume that $F$ is an algebraic number field of degree 4 with signature 2 and discriminant $d_{F}$, with $\left|d_{F}\right|<10^{6}$, and that $F$ contains a quadratic subfield $K$ with discriminant $d_{K}$. Since $F$ has a real embedding, $d_{K}>0$.

Let $\omega, \omega^{\prime}=\frac{1}{2}\left(\sigma \pm \sqrt{d_{K}}\right)$, where $\sigma \in\{0,1\}, \sigma \equiv d_{K}(\bmod 4)$, so that $K=\mathbb{Q}(\omega)$, and $\mathscr{O}_{K}=\mathbb{Z}[\omega]$ is the ring of integers of $K$.

We take $\rho \in \mathscr{O}_{F} \mid \mathscr{O}_{K}$, and let

$$
g(x)=x^{2}-\alpha x+\beta \in \mathscr{O}_{K}[x]
$$

be the minimal polynomial of $\rho$ over $K$, with

$$
\begin{aligned}
\alpha & =a_{1}+a_{2} \omega, & \beta & =b_{1}+b_{2} \omega, \\
\alpha^{\prime} & =a_{1}+a_{2} \omega^{\prime}, & \beta^{\prime} & =b_{1}+b_{2} \omega^{\prime} .
\end{aligned}
$$

Then the characteristic polynomial of $\rho$ over $\mathbb{Q}$ is

$$
\text { (9) } \begin{aligned}
f(x) & =\left(x^{2}-\left(a_{1}+a_{2} \omega\right) x+\left(b_{1}+b_{2} \omega\right)\right)\left(x^{2}-\left(a_{1}+a_{2} \omega^{\prime}\right) x+\left(b_{1}+b_{2} \omega^{\prime}\right)\right) \\
& =\left(x^{2}-\alpha x+\beta\right)\left(x^{2}-\alpha^{\prime} x+\beta^{\prime}\right) \\
& =x^{4}-s x^{3}+p x^{2}-q x+n \in \mathbb{Z}[x] .
\end{aligned}
$$

We define the $\mathbb{Z}$-module homomorphism $\lambda: \mathscr{O}_{F} \longrightarrow \mathbb{R}^{4}$ by

$$
\lambda(\xi)=\left(\frac{1}{2}\left(\xi_{1}-\xi_{2}\right), \frac{1}{2}\left(\xi_{2}-\xi_{1}\right), 0, \Im \xi_{3}\right)
$$

where $\xi_{1}, \xi_{2}$, are the real conjugates of $\xi$, and $\xi_{3}, \bar{\xi}_{3}$ the complex conjugates. Then $\lambda\left(\mathscr{O}_{F}\right)$ is a 2-dimensional lattice of determinant $\Delta=\sqrt{\left|d_{F}\right| / 8 d_{K}}$, and the kernel of $\lambda$ is $\mathscr{O}_{K}$. We choose $\rho \in \mathscr{O}_{F}$ so that $\|\lambda(\rho)\|^{2} \leq 2 \Delta / \sqrt{3}=\sqrt{\left|d_{F}\right| / 6 d_{K}}$ and $\operatorname{Tr}_{F / K}(\rho)=\alpha \in\{0+\omega, 1+\omega, 0+2 \omega, 1+2 \omega\}$. (Taking $\alpha \notin \mathbb{Q}$ ensures that $\rho$ has degree 4 over $\mathbb{Q}$.)

Since $\alpha$ and $\beta$ are real, we have

$$
\begin{aligned}
\alpha & =\rho_{1}+\rho_{2}, & \beta & =\rho_{1} \rho_{2}, \\
\alpha^{\prime} & =\rho_{3}+\bar{\rho}_{3}=2 \Re \rho_{3}, & \beta^{\prime} & =\rho_{3} \bar{\rho}_{3},=\left|\rho_{3}\right|^{2} .
\end{aligned}
$$

Therefore,

$$
\begin{aligned}
& \beta+\beta^{\prime}=\frac{1}{4}\left(\alpha^{2}+\alpha^{\prime 2}\right)-\frac{1}{4}\left(\rho_{1}-\rho_{2}\right)^{2}+\Im \rho_{3}{ }^{2}, \\
& \beta-\beta^{\prime}=\frac{1}{4}\left(\alpha^{2}-\alpha^{\prime 2}\right)-\frac{1}{4}\left(\rho_{1}-\rho_{2}\right)^{2}-\Im \rho_{3}{ }^{2},
\end{aligned}
$$

so that

$$
\begin{aligned}
\left|\left(\beta+\beta^{\prime}\right)-\frac{1}{4}\left(\alpha^{2}+\alpha^{\prime 2}\right)\right| & \leq \frac{1}{4}\left(\rho_{1}-\rho_{2}\right)^{2}+\Im \rho_{3}^{2} \leq\|\lambda(\rho)\|^{2}, \\
0 & \leq \frac{1}{4}\left(\alpha^{2}-\alpha^{\prime 2}\right)-\left(\beta-\beta^{\prime}\right) \leq\|\lambda(\rho)\|^{2} .
\end{aligned}
$$

We define $A_{1}=4 a_{1}^{2}+4 a_{1} a_{2} \sigma+a_{2}^{2}\left(d_{K}+\sigma\right), A_{2}=a_{2}\left(2 a_{1}+a_{2} \sigma\right)$, so that

It follows that

$$
\begin{aligned}
& \alpha^{2}+\alpha^{\prime 2}=\frac{1}{2} A_{1}, \quad \beta+\beta^{\prime}=2 b_{1}+b_{2} \sigma, \\
& \alpha^{2}-\alpha^{\prime 2}=A_{2} \sqrt{d_{K}}, \quad \beta-\beta^{\prime}=b_{2} \sqrt{d_{K}} \text {. }
\end{aligned}
$$

$$
\begin{aligned}
\frac{A_{2}-M}{4} & \leq b_{2} \leq \frac{A_{2}}{4}, \\
\frac{A_{1}-8 b_{2} \sigma-N}{16} & \leq b_{1} \leq \frac{A_{1}-8 b_{2} \sigma+N}{16},
\end{aligned}
$$


where

$$
M=\left\lfloor 4 \sqrt{\left|d_{F}\right| / 6\left|d_{K}\right|^{2}}\right\rfloor, \quad N=\left\lfloor 8 \sqrt{\left|d_{F}\right| / 6\left|d_{K}\right|}\right\rfloor .
$$

We define $G_{2}\left(d_{F}, d_{K}\right)$ to be the set of all polynomials $f(x) \in \mathbb{Z}[x]$ defined by (9), with $a_{1} \in\{0,1\}, a_{2} \in\{1,2\}$, and $b_{1}, b_{2}$ satisfying (10), (11).

Proposition 3. If $F$ is an algebraic number field of degree 4 and signature 2 with discriminant $d_{F}$ and ring of integers $\mathscr{O}_{F}$, and if $F$ contains a quadratic subfield $K$ with discriminant $d_{K}$ and ring of integers $\mathscr{O}_{K}$, then there exists $\rho \in \mathscr{O}_{F} \backslash \mathscr{O}_{K}$ with characteristic polynomial belonging to $G_{2}\left(d_{F}, d_{K}\right)$, such that $F=\mathbb{Q}(\rho)$.

\section{FIELdS WITH SMALl QUADRATIC INTEGERS}

Let $F$ be a quartic number field of signature 2 with discriminant $d_{F}>-10^{6}$. Suppose there exists $\rho \in \mathscr{O}_{F} \backslash \mathbb{Z}$ with characteristic polynomial in $G_{1}\left(d_{F}\right)$ such that $K=\mathbb{Q}(\rho)$ is a quadratic subfield of $F$. It follows from Proposition 2, using (5)-(8) with $d=-10^{6}$, that $0<d_{K}<80$.

For each quadratic field discriminant $d_{K}$ with $0<d_{K}<80$ we generate the polynomials in $G_{2}\left(-10^{6}, d_{K}\right)$ according to (9), taking $a_{1} \in\{0,1\}, a_{2} \in$ $\{1,2\}$, and $b_{1}, b_{2}$ running through the values specified by (10) and (11). For each such polynomial $f(x)$ we perform the following tests:

1. If $f(x)$ is reducible in $\mathbb{Z}[x]$, we exclude $f$.

2. If $f(x)$ is irreducible but $\mathbb{Q}(\rho)$ is not of signature 2 , we exclude $f$.

3. We compute the discriminant $d_{F}$ of the field $F=\mathbb{Q}(\rho)$.

4. If $\left|d_{F}\right| \geq 10^{6}$, we exclude $f$.

5. If $f \notin G_{2}\left(d_{F}, d_{K}\right)$, we exclude $f$.

Among the polynomials surviving these tests is a generating polynomial for every quartic number field of signature 2 with absolute discriminant less than $10^{6}$, such that no $\rho \in \mathscr{O}_{F} \backslash \mathbb{Z}$ with characteristic polynomial in $G_{1}\left(d_{F}\right)$ is a quartic integer.

\section{DETERMINING FIELD ISOMORPHISM}

Let $f$ and $g$ be irreducible monic quartic polynomials in $\mathbb{Z}[x], \alpha$ and $\beta$ roots of $f$ and $g$, respectively, $p$ a rational prime not dividing $D_{f}$ or $D_{g}$, and $n_{f}$ and $n_{g}$ the number of solutions in $\mathbb{Z} / p \mathbb{Z}$ to the congruences $f(x) \equiv 0$ $(\bmod p)$ and $g(x) \equiv 0(\bmod p)$. From Hensel's Lemma we know that $n_{f}$ and $n_{g}$ give the number of roots of $f$ and $g$ in $\mathbb{Z}_{p}$.

The fields $\mathbb{Q}[\alpha]$ and $\mathbb{Q}[\beta]$ are isomorphic if and only if there is a polynomial $h \in \mathbb{Q}[x]$ such that $h(\alpha)$ has characteristic polynomial $g$. If $\mathbb{Q}[\alpha]$ and $\mathbb{Q}[\beta]$ are isomorphic, then any root of $f$ lying in $\mathbb{Z}_{p}$ is taken by $h$ to a root in $\mathbb{Z}_{p}$ of $g$. It follows that $f$ and $g$ have the same number of roots in $\mathbb{Z}_{p}$, and that $h$ gives a one-to-one correspondence between them.

It is well known [3, Theorem 1], [10] that $n_{f}>0$ for infinitely many choices of $p$. We assume therefore that $p$ has been chosen so that $n_{f}=n_{g}>0$, and take $\alpha$ and $\beta$ to lie in $\mathbb{Z}_{p}$. Suppose

$$
\lambda_{0}+\lambda_{1} \alpha+\lambda_{2} \alpha^{2}+\lambda_{3} \alpha^{3}+\lambda_{4} \beta=0, \quad \lambda_{j} \in \mathbb{Z}_{p}, 0 \leq j \leq 4 .
$$

The rational solutions of (12) form a $\mathbb{Z}$-lattice of rank at most 1 (otherwise 1 , $\alpha, \alpha^{2}, \alpha^{3}$ would be dependent over $\mathbb{Q}$ ). The $p$-adic solutions of (12) form a $\mathbb{Z}_{p}$-lattice of rank 4 , with $\mathbb{Z}_{p}$-basis given by the columns 
Consider now the relation

$\begin{array}{cccc}-\alpha & -\alpha^{2} & -\alpha^{3} & -\beta \\ 1 & 0 & 0 & 0 \\ 0 & 1 & 0 & 0 \\ 0 & 0 & 1 & 0 \\ 0 & 0 & 0 & 1\end{array}$

$$
\lambda_{0}+\lambda_{1} \alpha+\lambda_{2} \alpha^{2}+\lambda_{3} \alpha^{3}+\lambda_{4} \beta \equiv 0 \quad\left(\bmod p^{m}\right) .
$$

The rational solutions of (13) form a $\mathbb{Z}$-lattice $L_{m}$ of rank 5 , with $\mathbb{Z}$-basis given by the columns

$\begin{array}{ccccc}p^{m} & -\bar{\alpha} & -\bar{\alpha}^{2} & -\bar{\alpha}^{3} & -\bar{\beta} \\ 0 & 1 & 0 & 0 & 0 \\ 0 & 0 & 1 & 0 & 0 \\ 0 & 0 & 0 & 1 & 0 \\ 0 & 0 & 0 & 0 & 1\end{array}$

where $\bar{\alpha}, \bar{\beta}$ are rational approximations to $\alpha, \beta$, correct modulo $p^{m}$. ( $\bar{\alpha}$ and $\bar{\beta}$ are easily computed by Hensel-Newton lifting from roots modulo $p$ of $f$ and $g$.)

If a nontrivial rational solution of (12) exists, it can be shown (see [6]) that for sufficiently large $m$ it appears as a short vector in the lattice $L_{m}$, and is therefore accessible via lattice basis reduction techniques (see [1, 7]).

It is known that the effectiveness of basis reduction depends upon, and is predictable from, the size of the entries. For these computations we found a choice of $m$ such that $p^{m} \approx 10^{25}$ to be effective.

\section{RESULTS}

It should be noted to begin with, that our results are in complete agreement with the work of Godwin [4, 5]. The distribution of Galois groups by field discriminant appears in the appendix (cf. $[1,2])$.

The main computations were done on a Digital Equipment VAX 8550 computer at the Computer Centre of Concordia University. Statistics for the totally real and totally complex cases (using the most recent versions of the software) are included for comparison. Execution times are expressed in CPU-hours.

Signature 0: 104:12 CPU-hours, 81322 fields.

Signature 2: 222:15 CPU-hours, 90671 fields.

Signature 4: 5:30 CPU-hours, 13073 fields.

The following data has been prepared for each of the fields (all signatures):

- generating polynomial

- field discriminant

- Galois group

- integral basis

- quadratic subfield discriminants (imprimitive fields only)

- fundamental units

- class group

The computation of fundamental units and class groups was done with KANT on Hewlett-Packard Apollo workstations at Düsseldorf. The data is available on magnetic media from the authors. 


\section{APPENDIX}

\section{Distribution of Galois groups by field discriminant}

\begin{tabular}{|c|c|c|c|c|}
\hline & $\mathrm{D}_{8}$ & & $\mathrm{~S}_{4}$ & \\
\hline 0 : & 193 & $\|||||||||||$ & 822 & ||||||||||||||||||||||||||||||||||||||||||||||||||||| \\
\hline 20000: & 190 & iliilililiil & 1144 & 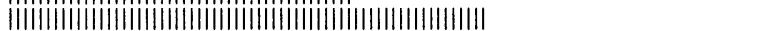 \\
\hline 40000: & 204 & iliiliiliiil & 1236 & 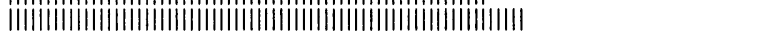 \\
\hline 60000: & 186 & IIIIIIIIII & 1359 & 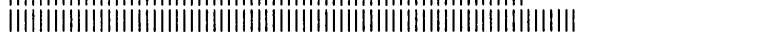 \\
\hline 80000: & 195 & iimiliili. & 1355 & 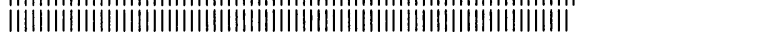 \\
\hline 100000: & 187 & IIIIIIII" & 1419 & 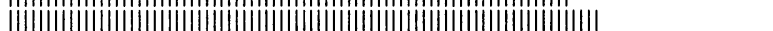 \\
\hline 120000: & 195 & IIiiiliili|i| & 1402 & 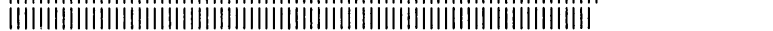 \\
\hline 140000: & 212 & 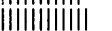 & 1457 & 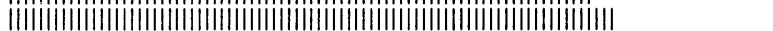 \\
\hline 160000: & 224 & IIIIIIIIIII & 1544 & 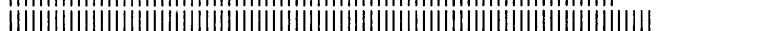 \\
\hline 180000: & 181 & IIIIIIIIII| & 1513 & 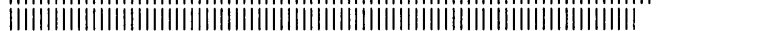 \\
\hline 200000: & 179 & IIIIIIIIII & 1534 & 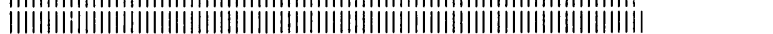 \\
\hline 220000: & 179 & IIIIIIiili & 1459 & 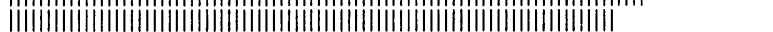 \\
\hline 240000: & 222 & 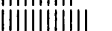 & 1531 & 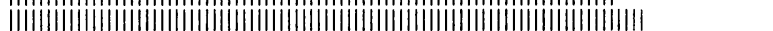 \\
\hline 260000: & 195 & IIIIIIIIIII & 1663 & 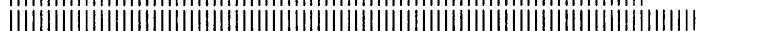 \\
\hline 280000: & 186 & IIIIIIIIi" & 1582 & 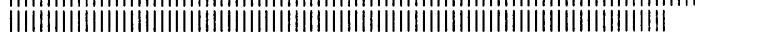 \\
\hline 300000: & 209 & IIIIIIIIII|| & 1620 & 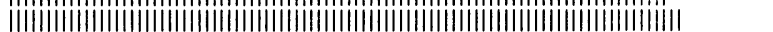 \\
\hline 320000: & 219 & IIIIIIIIIII & 1505 & 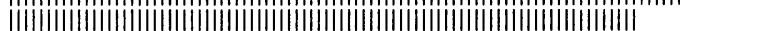 \\
\hline 340000: & 190 & IIIIIIIIIIII & 1635 & 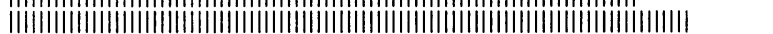 \\
\hline 360000: & 180 & IIIIIIIII" & 1636 & м1" \\
\hline 380000: & 190 & Iiliiliilili & 1647 & 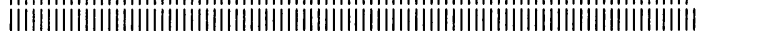 \\
\hline 400000: & 176 & "IIIIIIIIII & 1634 & 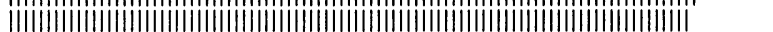 \\
\hline 420000: & 197 & 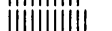 & 1658 & 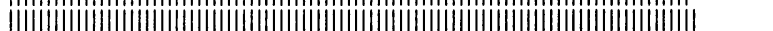 \\
\hline 440000: & 195 & |ilifilililii| & 1649 & 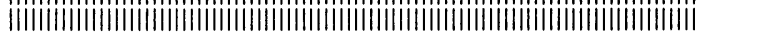 \\
\hline 460000: & 188 & IIIIIIIIIIIi| & 1637 & 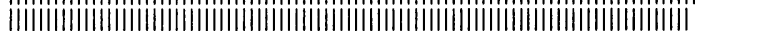 \\
\hline 480000: & 193 & 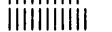 & 1628 & мuा \\
\hline 500000: & 214 & mimimim & 1646 & 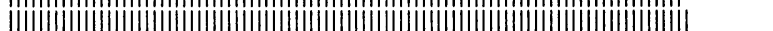 \\
\hline $520000:$ & 187 & "IIIIIIII!" & 1708 & 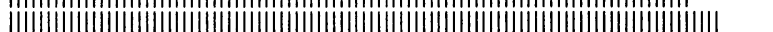 \\
\hline 540000: & 196 & IIIIIIIIi! & 1675 & 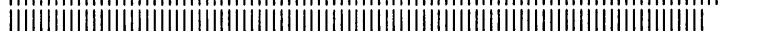 \\
\hline 560000: & 193 & ifilifilifili & 1733 & 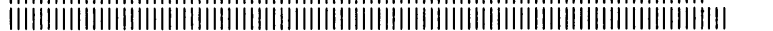 \\
\hline 580000: & 196 & IIIIIIIiilili & 1696 & 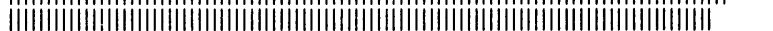 \\
\hline 600000: & 188 & Iiliiimili" & 1764 & 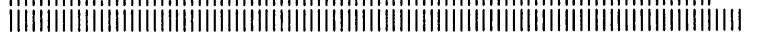 \\
\hline 620000: & 184 & Iiliiiiliili & 1753 & 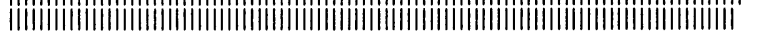 \\
\hline 640000: & 206 & iliiiiliili| & 1726 & 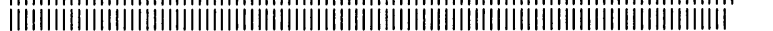 \\
\hline 660000: & 200 & IIIIIIIIIIII & 1648 & 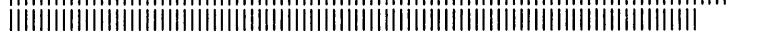 \\
\hline 680000: & 197 & Iiiilili|iil & 1745 & 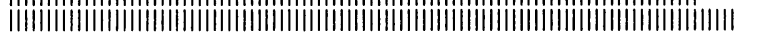 \\
\hline 700000: & 201 & Iiliililili & 1737 & 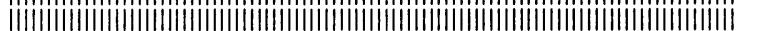 \\
\hline 720000: & 211 & IIIIIIIIII & 1762 & 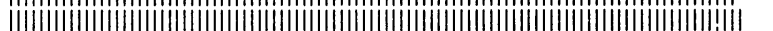 \\
\hline 740000: & 185 & iiiliiliain & 1827 & 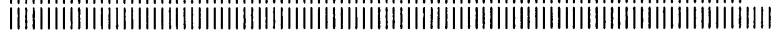 \\
\hline 760000: & 185 & Iiliाiiliii & 1799 & 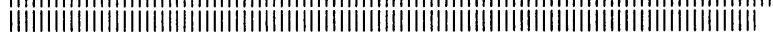 \\
\hline $780000:$ & 195 & Iiliiiili:i & 1762 & 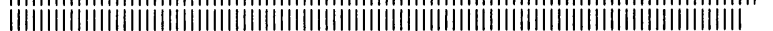 \\
\hline 800000: & 215 & 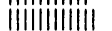 & 1726 & 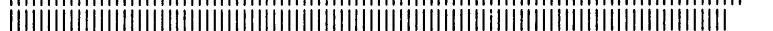 \\
\hline 820000: & 226 & IIIIIIiiliilin & 1750 & 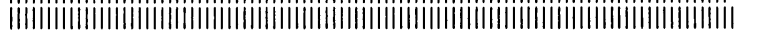 \\
\hline $840000:$ & 180 & IIIIIIIII & 1740 & 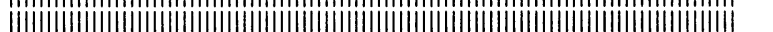 \\
\hline 860000: & 213 & 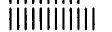 & 1758 & 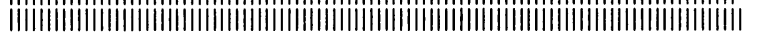 \\
\hline 880000: & 184 & "iinimili" & 1810 & 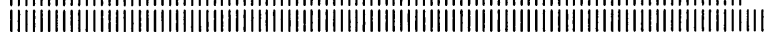 \\
\hline 900000: & 188 & milimimi & 1786 & 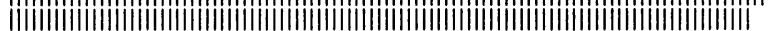 \\
\hline 920000: & 193 & IIIIIIIIIIII & 1719 & 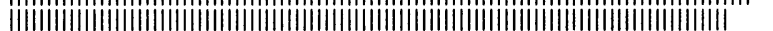 \\
\hline 940000: & 198 & 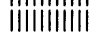 & 1806 & 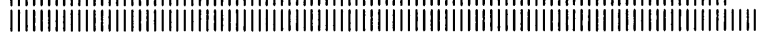 \\
\hline 960000: & 194 & iilifilifilili & 1749 & 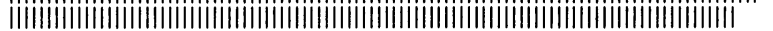 \\
\hline 980000: & 178 & "IIIIIIIIII & 1805 & 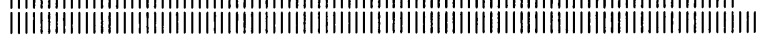 \\
\hline Total: & 9772 & & 80899 & \\
\hline Percent: & 10.78 & & 89.22 & \\
\hline
\end{tabular}

\section{BIBLIOGRAPHY}

1. J. Buchmann and D. Ford, On the computation of totally real quartic fields of small discriminant, Math. Comp. 52 (1989), 161-174.

2. D. Ford, Enumeration of totally complex quartic fields of small discriminant, Computational Number Theory, Proceedings of the Colloquium on Computational Number Theory, Debrecen (Hungary), 1989 (A. Pethö, M. Pohst, H. C. Williams, and H. G. Zimmer, eds.), de Gruyter, Berlin and New York, 1991, pp. 129-138.

3. I. Gerst and J. Brillhart, On the prime divisors of a polynomial, Amer. Math. Monthly 78 (1971), 250-266. 
4. H. J. Godwin, On quartic fields of signature one with small discriminant, Quart. J. Math. Oxford Ser. (2) 8 (1957), 214-222.

5. __ On quartic fields of signature one with small discriminant. II, Math. Comp. 42 (1984), 707-711.

6. A. K. Lenstra, Lattices and factorization of polynomials over algebraic number fields, Proceedings Eurocam 82, Lecture Notes in Comput. Sci., vol. 144, Springer, Berlin, 1982, pp. 32-39.

7. A. K. Lenstra, H. W. Lenstra Jr., and L. Lovasz, Factoring polynomials with rational coefficients, Math. Ann. 261 (1982), 515-534.

8. M. Pohst, On the computation of number fields of small discriminants including the minimum discriminants of sixth degree fields, J. Number Theory 14 (1982), 99-117.

9. __ On computing isomorphisms of equation orders, Math. Comp. 48 (1987), 309-314.

10. I. Schur, Über die Existenz unendlich vieler Primzahlen in einigen speziellen arithmetischen Progressionen, S.-B. Berlin Math. Ges. 11 (1912), 40-50.

Fachbereich 10, INFormatik, Universität des SAARlandes, SAarbrücken, Germany E-mail address: buchmann@cs.uni-sb.de

Department of Computer Science, Concordia University, Montréal, Canada

E-mail address: kbkfe24@vax2.concordia.ca

Mathematisches Institut, HeinRICh-Heine-Universität, DüSSEldoRf, GeRMaNy

E-mail address: pohst@ze8.rz.uni-duesseldorf.de 